\title{
Equine Syndromic Surveillance in Colorado Using Veterinary Laboratory Test Order Data
}

\author{
Howard Burkom¹, Yevgeniy Elbert ${ }^{1}$, Jerrold Dietz, Barbara Powers², Cynthia \\ Johnson $^{3}$, Leah Estberg ${ }^{3}$ and Judy Akkina ${ }^{3}$
}

'Johns Hopkins Applied Physics Laboratory, Laurel, MD, USA; ${ }^{2}$ Vet Teaching Hospital, Colorado State University, Fort Collins, CO, USA; ${ }^{3}$ Center for Epidemiological and Animal Health, US Dept. of Agriculture, Fort Collins, CO, USA

\section{Objective}

Establish a laboratory-based syndromic surveillance system for horses in Colorado.

\section{Introduction}

The Risk Identification Unit (RIU) of the US Dept. of Agriculture's Center for Epidemiology and Animal Health (CEAH) conducts weekly surveillance of national livestock health data and routine coordination with agricultural stakeholders. In an initiative to increase the monitored species, health issues, and data sources, CEAH epidemiologists are building a surveillance system based on weekly counts of laboratory test orders along with Colorado State Univ. laboratorians and statistical analysts from the Johns Hopkins Univ. Applied Physics Lab. Initial efforts used 12 years of equine test records from 3 state labs covering most Colorado horse testing. Trial syndrome groups were formed based on RIU experience and published articles ${ }^{1}$. Data analysis, stakeholder input, and discovery of laboratory workflow details were needed to modify these groups and filter test records to eliminate alerting bias. Customized statistical monitoring methods were sought based on specialized lab information characteristics and on likely presentation and health significance of syndrome-associated diseases.

\section{Methods}

Data transformation and syndrome formation focused on test names, order completion status, submitting organization, specimen type, analyte measured, animal owner zip code, and lab unit that received the order. We analyzed time series of weekly counts of tests included in candidate syndrome groups and conducted an iterative process of data analysis and veterinary consultation for syndrome refinement and record filters. This process produced a rule set in which records were .classified into syndromes using only test name when possible and otherwise, the specimen type or related body system was used with test name to determine the syndrome. Test orders associated with government regulatory programs, veterinary teaching hospital inpatient testing protocols, or research projects, rather than clinical concerns, were excluded.

We constructed a testbed for sets of 1000 statistical trials and applied a stochastic injection process assuming lognormally distributed incubation periods to choose an alerting algorithm with the syndrome-required sensitivity and an alert rate within the specified acceptable range for each resulting syndrome. Alerting performance of the EARS C3 algorithm traditionally used by CEAH was compared to modified C2, CuSUM, and EWMA methods, with and without outlier removal and adjustments for the total weekly number of nonmandatory tests.

\section{Results}

The equine syndrome groups adopted for monitoring were abortion/ reproductive, diarrhea/GI, necropsy, neurological, respiratory, systemic fungal, and tickborne. Data scales and seasonality differed widely among syndrome series. Removal of mandatory tests reduced weekly observed counts by up to $80 \%$. The RIU group studied outcomes associated with each syndrome and called for detection of single-week signals for most syndromes with expected false-alert intervals $>8$ and $<52$ weeks, 8 -week signals for neurological and tickborne monitoring (requiring enhanced sensitivity), 6-week signals for respiratory, and 4-week signals for systemic fungal. From the test-bed trials, recommended methods, settings and thresholds were derived. Figure 1 shows a performance comparison based on 8-week signals for the neurological time series.

\section{Conclusions}

Understanding of laboratory submission sources, laboratory workflow, and of syndrome-related outcomes are crucial to form syndrome groups for routine monitoring without artifactual alerting. Choices of methods, parameters, and thresholds varied by syndrome and depended strongly on veterinary epidemiologist-specified performance requirements.

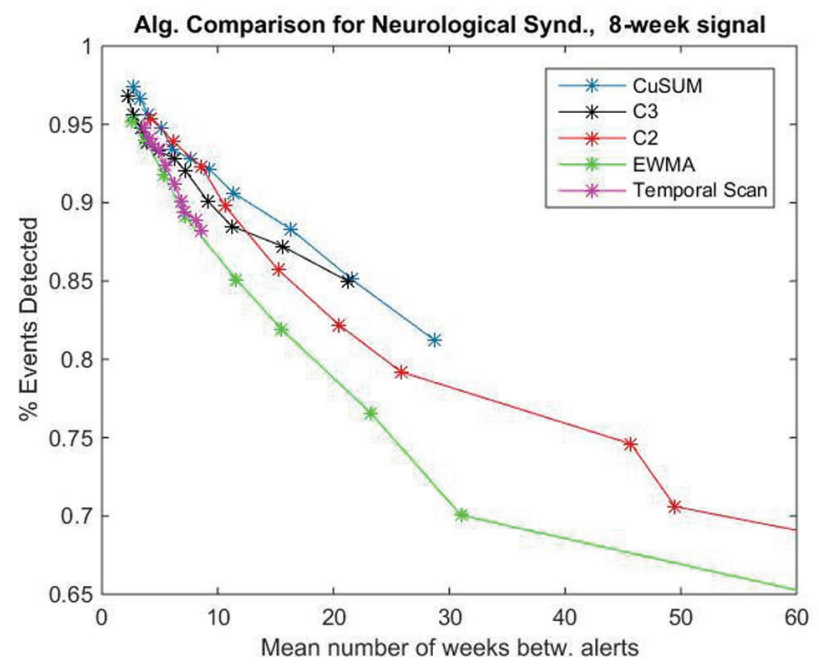

Algorithm Performance Comparison for Neurological Syndrome

\section{Keywords}

livestock surveillance; veterinary syndrome; alerting algorithm; animal health; detection performance

\section{References}

Dorea FC, Muckle CA, Kelton D, et al. Exploratory analysis of methods for automated classification of laboratory test orders into syndromic groups in veterinary medicine. PLoS One. Vol 8. United States2013:e57334.

\section{*Howard Burkom}

E-mail: howard.burkom@jhuapl.edu 